\title{
¿Inflando el "Glovo"?: Un análisis sobre los nuevos mecanismos de delivery y su abordaje en la jurisprudencia reciente
}

Inflating the "Glove"?: An analysis on the new mechanisms of delivery and its treatment in the recent jurisprudence

¿En gonflant le "Glovo"? Une analyse des nouveaux mécanismes de délivrance et de son approche dans la jurisprudence récente

¿Inflando o “Glovo"?: Uma análise sobre os novos mecanismos de entrega e sua abordagem na jurisprudência recente

Lucio A. Vallefin ${ }^{1}$ Universidad Nacional de La Plata

Revista Derechos en Acción

Año 3/No 9 Primavera 2018, 403-426

DOI: https://doi.org/10.24215/25251678e229

ORCID: https://orcid.org/0000-0003-1330-5367

Recibido: 15/06/2018

Recibido con modificaciones: 8/09/2018

Aprobado: 12/11/2018

Resumen: El siguiente trabajo tiene como finalidad realizar un breve análisis sobre la recepción que ha tenido el fenómeno empresarial Ilamado "Economía colaborativa" dentro del marco del Derecho del Trabajo. Para tal fin, se tomará como objeto de estudio a una de las empresas de este tipo con mayor inserción en el rubro del envío a domicilio de productos en nuestro país, la española Glovo, analizando la situación en la que se enmarca la relación que llevan adelante con quienes realizan sus envíos (Glovers) a la luz de la jurisprudencia reciente.

\footnotetext{
1 Abogado (UNLP). Becario en Centro de Investigación de Derecho Crítico de la Facultad de Ciencias Jurídicas y Sociales (en trámite de aprobación formal). UNLP. Colaborador en Cátedra III de Derecho Social de la Facultad de Ciencias Jurídicas y Sociales. UNLP
} 
Palabras clave: Glovo - Economía colaborativa - Derecho laboral - Jurisprudencia.

Resumo: Este trabalho tem como objetivo fazer uma breve análise sobre a recepção que o fenômeno empresarial denominado "Economia Colaborativa" teve no âmbito do Direito do Trabalho. Para tanto, será tomada como objeto de estudo uma das empresas desse tipo com maior inserção na categoria de entrega em domicilio de produtos em nosso país, a empresa espanhola Glovo, analisando a situação na que se enquadra a relação que levam adiante com aqueles que fazem suas entregas (Glovers), à luz da jurisprudência recente.

Palavras-chave: Glovo - Economia colaborativa - Direito do trabalho Jurisprudência

Abstract: The following work has the purpose of making a brief analysis about the reception that the business phenomenon called "Collaborative Economy" has had within the framework of the Labor Law. For this purpose, one of the companies of this type with greater insertion in the area of product delivery in our country, the Spanish company Glovo, will be taken as our object of study, analyzing the situation in which their relationship with those who make their deliveries (Glovers) is framed, in light of the recent jurisprudence.

Key words: Glovo. Collaborative economy. Labor Law. Jurisprudence.

Résumé: L'objectif de ce travail est la réalisation d'un bref analyse sur la perception du phénomène entrepreneurial appelé "économie collaborative" dans le droit du travail. Dans ce but, on prendra comme objet de notre analyse l'une des entreprises de ce type ayant la plus grande insertion dans la catégorie de livraison à domicile de produits dans notre pays, l'espagnole "Glovo", en analysant la situation dans laquelle s'encadre la relation qu'elles entretiennent avec ceux qui font leurs livraisons (Glovers) à la lumière de la jurisprudence récente.

Mots-cles: Glovo, économie collaborative, droit du travail, jurisprudence.

\section{Introducción}

Recientemente se ha podido ver en Argentina, especialmente en grandes núcleos urbanos como Rosario, La Plata y 
Capital Federal, el desarrollo y crecimiento de diversos sistemas de entrega de productos a domicilio por medio de aplicaciones para smartphones (teléfonos con acceso a internet). A la que podría considerarse como pionera de esta modalidad en el país, la uruguaya PedidosYa, se han sumado recientemente otras, entre las que puede destacarse, por su repentino crecimiento en el país, a la española Glovo.

Esta última, a la cual se presenta como una aplicación (app) que te permite pedir "los mejores productos en tu ciudad", es una empresa fundada en el año 2015 en Barcelona, España, que basa su sistema de envíos en la posibilidad de solicitar productos, por medio de una aplicación para celulares, con un amplio margen de elección en cuanto a lo solicitado (estableciéndose solamente un límite en cuanto al tamaño y peso del producto y la imposibilidad de transportar animales). La nota distintiva entre el servicio ofrecido por esta app y similares (siendo Rappi la más reconocida, por su amplia actividad en Capital Federal) y el ya mencionado PedidosYa, quien anteriormente operaba en esos mismos términos, es la relación que establecen con la persona que se encarga de realizar el pedido, siendo en este último reconocida como una relación de trabajo (con sus propias regulaciones), mientras que en el resto, se ha denominado a esta figura bajo el rótulo de "colaboradores", lo cual ha generado diversas polémicas que van a ser abordadas en el presente trabajo.

Glovo opera desde una lógica de negocios y actividad empresarial con un origen muy reciente, conocida como "economía colaborativa", este concepto todavía no ha tenido un gran desarrollo teórico en nuestro país, pero se puede apreciar un creciente impacto del mismo en las transformaciones del mercado de trabajo. La realidad es que este concepto está en desarrollo y es esencialmente una estrategia discursiva, la cual vive en constante tensión con fenómenos del ámbito laboral como lo son la tercerización y el fraude. El objetivo de los siguientes apartados es explorar estas ideas y ver cómo las mismas han sido receptadas por la jurisprudencia y la normativa, para lo cual va acudirse tanto a resoluciones referentes a la misma Glovo, 
como a otros emprendimientos, tanto del mismo rubro, como de otros que utilicen mecánicas tecnológicas similares (siendo el caso más relevante el de Uber).

\section{II. ¿Qué es la economía colaborativa? Visiones comparadas sobre la actividad desarrollada por Glovo}

Para abordar el concepto de "economía colaborativa", en primer lugar, hay que tener en cuenta que el mismo no es definible con total consenso, ya que, más allá de ser un objeto de estudio teórico, resulta una construcción que busca englobar variedad de fenómenos y actividades que no tienen mayor conexión que la modalidad en la que se comercializan y distribuyen. Por lo dicho, como señala Pacheco Jimenez, antes de abordar este objeto de estudio, es necesario partir de la premisa de la Web 2.0., la cual supone una reestructuración en la forma en la que opera la comunicación e interacción por internet.

La Web 2.0. se entiende como una "tendencia basada el desarrollo de diversas aplicaciones en Internet, posibilitando la interacción con el resto de los usuarios y aportando contenido actualizado" 2 , entendiéndose como una superación a la idea original de Web 1.0., la cual operaba con el usuario como un sujeto pasivo, únicamente receptor de información. A partir de esta noción, es que surgen los denominados Negocios 2.0. (también llamados negocios vía web u online), los cuales aprovechan esta faceta de intercomunicación para realizar transacciones y operaciones comerciales sin tener que mediar en un ámbito físico, lo cual permite a su vez ajustarse a diversidad de operadores (desde el mercado financiero hasta los pequeños consumidores).

Dentro de esta idea es que opera la ya mencionada "Economía colaborativa", la cual puede definirse, según la investigadora

2 PACHECO JIMÉNEZ, MARÍA NIEVES. La Web 2.0 como instrumento esencial en la economía colaborativa: Auge de negocios de dudosa legalidad. Publicado en Revista CESCO de Derechos de Consumo N¹7/2016 - Universidad de Castilla y La Mancha, España 
previamente citada, como "aquella manera de compartir o intercambiar tanto bienes tangibles como intangibles (tiempo, espacio, hobbies) a través de la tecnología moderna y las comunidades sociales". Esta noción, extremadamente amplia en un primer acercamiento, puede delimitarse al entender que no toda actividad comercial realizada en la Web 2.0. es Economía colaborativa, sino que estas actividades requieren necesariamente del uso de una plataforma con acceso a internet (en la práctica, esencialmente, aplicaciones para celular) para poder concretarse o materializarse a la realidad, funcionando la misma como organizador y habilitador, lo cual marca la diferencia con un comercio on line - el cual, en caso de no tener esa plataforma, puede seguir operando en un ámbito físico, siendo la web una simple herramienta -

En este trabajo se abordará un modelo de economía colaborativa basado en la intervención de terceros que se encargan de la realización de los servicios ofrecidos por la empresa, sin formar parte formalmente de su estructura. Este fenómeno no ha tenido una denominación clara, siendo llamado por algunos, entre otros nombres, "Gig economy" (economía de los trabajos temporales) y por otros "Trabajo en economía de plataformas" (término acuñado por la OIT). Esta imprecisión se debe, fundamentalmente, al conflicto que estas actividades tienen con respecto a la estructura tradicional del mercado de trabajo, generando, en conjunto con otros factores, una reciente preocupación sobre la dirección del futuro del mismo reconocida por actores de gran relevancia -entre los que destaca la Organización Internacional del Trabajo (OIT, ILO en inglés)-, a la vez que una gran promoción de la misma por múltiples empresas, quienes la están empezando a ver como una forma de reducir costos e impulsar sus proyectos dentro del mercado ${ }^{3}$.

\footnotetext{
3 Uno de los ejemplos más cuestionados ha sido el proyecto Amazon Flex, en el cual la empresa de mensajería Amazon utiliza a repartidores externos, siendo recientemente investigada como fraudulenta por el Ministerio de Trabajo de España. Vease: http://www.ticbeat. com/empresa-b2b/trabajo-investiga-amazon-reparto-paquetes-particulares/
} 
Finalmente, tras este contexto, es que se puede señalar, dentro de este tipo de trabajos una distinción entre las mecánicas de las empresas que los utilizan, centrada esencialmente en el servicio que ofrecen. Por un lado, se encuentran aquellas que ofrecen un servicio directo a quien consume, entre las que se destaca Uber, empresa que ofrece transporte por conductores que se encuentran logueados en su sistema web ( $\sin$ formar parte de la empresa nominalmente), los cuales aportan sus propios vehículos para realizar la actividad. Por otro, se pueden señalar múltiples empresas que ofrecen a otras la tercerización ${ }^{4}$ de un servicio, entre las que destacan las que realizan recados, esencialmente en la modalidad delivery (envío de productos puerta a puerta), receptando los pedidos de los clientes y habilitando a terceros a operar a su nombre para realizar el reparto, dentro de estas últimas se encuentra la que va a ser nuestro objeto de estudio, Glovo.

Ya establecido el concepto, hay que dejar en claro que la economía colaborativa, como todo implemento tecnológico, no supone una práctica negativa de por sí, sino una herramienta, es así que se ha destacado incluso a la misma como un proyecto superador al propio sistema capitalista tradicional destacándose, "su carácter participativo, basado en las auténticas necesidades de los individuos y en la capacidad de acceso de éstos al nuevo sistema global de consumo, en oposición a la premisa de acumulación de bienes como un fin en sí mismo"' y su utilidad frente a momentos de crisis económica (especialmente tras la de 2008), señalando Doménech Pascual ${ }^{6}$ que "el deterioro de las

\footnotetext{
4 Según Lozano y Casiraghi, se entiende por Tercerización a la "tendencia empresarial y productiva a realizar parte de sus actividades a través de otras unidades más o menos independientes. Comprende todos los fenómenos de organización del trabajo en los cuales la empresa recurre a trabajadores, real o ficticiamente externos". LOZANO, MARÍA PAULA; CASIRAGHI, FEDERICO. Tercerización, precarización y solidaridad en las relaciones laborales. La Ley Online. Cita Online: AR/DOC/1477/2013

5 LO BOSCO, LUCIANA MARINA. El paradigma del consumo colaborativo y sus implicancias en las prácticas turísticas. Monografía de graduación presentada en marzo de 2017. Universidad Nacional de Mar del Plata. Disponible en: http://nulan.mdp.edu.ar/2729/1/lobosco-2017.pdf

6 DOMÉNECH PASCUAL, GABRIEL. La regulación de la economía colaborativa (El caso Uber contra Taxi). REVISTA CEFLEGAL. CEF, núms. 175-176 (agosto-septiembre 2015) - Pag. 67
} 
condiciones económicas en la mayoría de los países occidentales y las estrecheces resultantes han servido de potente estímulo para que mucha gente invente, desarrolle, ponga en marcha o participe en actividades de economía colaborativa". Pero, como toda herramienta, puede ser usada con otros fines, entre los que destaca la flexibilización laboral ${ }^{7}$, para la cual resulta ideal, según Vallecillo Gámez ${ }^{8}$ por tres factores: la división del trabajo en tareas independientes de corta duración (evitando creación de vínculos estables, a la vez que atrae a un público que busca actividades que no le exijan un tiempo excesivo de trabajo), la externalización del servicio a través de trabajadores/as "autónomos" (con lo que puede garantizarse una reducción de los costes y riesgos económicos), y el ahorro en tiempo y costes que supone para la ciudadanía, a lo cual se podría sumar un cuarto elemento, abordado por Verdolini ${ }^{9}$, el cual es la reducción de costes fiscales a causa de su difuminado margen de operaciones, el cual implica inserción en múltiples mercados y financiamientos que no dejan en claro en qué territorio se debe tributar.

En base a lo previamente dicho, se puede ver como Glovo resulta un gran ejemplo en cuanto a estos usos de la economía colaborativa. Oscar Pierre, joven empresario de 25 años, logró el despegue de su empresa incipiente (como se las denomina en la jerga empresarial, Start-Up) por medio de la vinculación con diversos grupos de captación de fondos y operadoras de capital de riesgo ${ }^{10}$, con los cuales ha podido tener acceso a

7 El concepto es definido por Zamboni como "(...) la parte de la política conservadora que tiene como objetivo la derogación máxima posible de la legislación que en su conjunto se conoce como Derecho del Trabajo y de la Seguridad Social". ZAMBONI, HORACIO - Flexibilidad laboral, desandar la historia. Cuadernos del Sur N 15 Abril 1993.

8 VALLECILLO GÁMEZ, MARÍA ROSA. Economía colaborativa y laboralidad: Los cabos sueltos entre el vacío legal y la dudosa legalidad. Propuesta para Conversación 3 sobre El futuro del trabajo organizada por OIT - Universidad de Jaén.

9 VERDOLINI, VALERIA S. Multinacionales en forma de app: ¿Innovación o evasión?. REDEA Revista Derechos en Acción, Año 2 №3, Otoño 2017

10 Se pueden destacar, entre otros, a Seaya Ventures y The Crowd Angel. Más información en: https://retina.elpais.com/retina/2017/12/19/innovacion/1513692348_055977.html y http://blog.thecrowdangel.com/blog/casos-de-exito-de-the-crowd-angel-glovo/ 
múltiples cadenas de restaurantes, locales de comida rápida, etc (a los cuales denomina partners), logrando una financiación de trescientos millones de euros en mayo ${ }^{11}$, los cuales se tradujeron en Argentina a sesenta mil pedidos por mes ${ }^{12}$. El éxito de la empresa ha sido atribuido por Pierre ${ }^{13}$ a la aplicación de prácticas flexibilizadoras como estrategia de negocios, es decir, dinámicas que funcionan con una regulación muy laxa, permitiéndoles introducirse en múltiples economías.

Tras sostener ese uso de la flexibilidad, en la misma entrevista se le preguntó si entiende a las relaciones que tiene la empresa con sus "Glovers" (terceros que se registran en la aplicación y proceden a realizar los envíos) como explotación, a lo que respondió "Te cuento qué es un Glover: mensajero con vehiculo propio y smartphone, autónomo validado, una aplicación que se descargan [dos euros] y a la que se conectan cuando quieran para aceptar los pedidos que elijan. Nosotros arbitramos lo minimo, nos limitamos a garantizar que los pedidos se hagan bien [control de calidad], lo mismo que arbitramos el market place y el usuario". Esta posición, que presenta a los mismos como terceros ajenos a la empresa encuentra una gran tensión incluso en las mismas palabras de su director, el cual luego señaló que "Para esta empresa, el glover es la pata más importante: si no bay mensajeros todo se cae". Esta incertidumbre en la situación de las personas que trabajan allí ha hecho que, a pesar de no ser percibido inicialmente, debido a lo incipiente de la práctica, este modelo de vinculación con las trabajadoras/es resulte cada vez más cuestionado, derivando en múltiples acciones sociales y políticas con un gran crecimiento impulsado, por un lado, en las primeras acciones judiciales españolas, y, por otro, en la fuerte inserción de estas modalidades en Latinoamérica.

\footnotetext{
11 En línea en: https://marketing4ecommerce.net/glovo-ronda-financiacion-expansioninternacional/

12 En línea en: https://pulsosocial.com/2018/05/02/glovo-Ilega-casi-al-millon-de-pedidosen-todos-sus-mercados-y-se-afianza-en-argentina/

13 En línea en: http://www.expansion.com/fueradeserie/personajes/2018/07/10/5b3b58382 2601d9d538b463f.html
} 
Según un Informe del Banco Interamericano de Desarrollo $^{14}$, Argentina, Brasil y México son los países con mayor número de iniciativas, detectando 107 startups de este tipo en la región. Esta inserción (en el caso argentino) es atribuida, según Carlos Correa, líder regional de comunicación de Rappi, al gran desarrollo de la cultura del delivery y a una "coyuntura politica mucho más adecuada para la inversión extranjera"15, a lo cual Matías Gath, el responsable nacional de Glovo agrega que "La gente está acostumbrada a enviar a otra persona a hacer mandados. Es el famoso 'che, pibe' que se encarga de distintos trámites". De los dichos antes citados puede extraerse una idea clara: La llegada al país de empresas como Glovo es producto de una flexibilización en las políticas de empleo, apoyada por una apertura económica hacia los inversores extranjeros, implicando una mayor tolerancia a figuras laborales impropias. Lo dicho puede verse en las reiteradas denuncias de incumplimiento normativo que este tipo de actividades ha recibido por actores como la CGT o ASIMM ${ }^{16}$, considerando que este modelo de "colaboradores autónomos" configura un caso de Fraude a la ley previsto por el artículo 14 de la Ley de Contrato de Trabajo (ley 20.744) ${ }^{17}$, la cual oculta una relación laboral, impidiendo la aplicación de la normativa vigente en materia de trabajadores/ as de mensajería (el Convenio Colectivo 722/15 y la Resolución CNC 604/2011, que crea el Subregistro de Prestadores de Servicios de Mensajería de la ENACOM, entre otras). Esta situación en la que se encuentran quienes desempeñan la actividad supone a su vez una manera de eludir responsabilidades laborales,

\footnotetext{
14 Economía Colaborativa en América Latina. Informe del Banco Interamericano de DesaroIlo (BID)

15 https://www.lanacion.com.ar/2118668-economia-colaborativa-la-revolucion-digitalllega-a-los-cadetes

16 Asociación Sindical de Motoristas y Mensajeros. http://www.asimm.org.ar/?p=2862

17 Art. 14. - Nulidad por fraude laboral. Será nulo todo contrato por el cual las partes hayan procedido con simulación o fraude a la ley laboral, sea aparentando normas contractuales no laborales, interposición de personas o de cualquier otro medio. En tal caso, la relación quedará regida por esta ley.
} 
tanto en lo que respecta los Accidentes de Trabajo (ya que al figurar como autónomas, las personas que operan en estos espacios no se encuentran incluidas en el régimen de la ley 24.557), como las derivadas de otros incumplimientos, caso de las indemnizaciones previstas por despido sin causa, resultando esto en un beneficio no solo para la empresa en cuestión, sino para aquellas que tercerizan su actividad, evitando responder en caso pretender una extensión solidaria a las mismas, debido al no reconocimiento de la relación inicial.

Frente a esta situación, hay que considerar que el derecho, como técnica que utiliza el orden social para motivar o evitar $\operatorname{conductas}^{18}$, puede ser una gran herramienta para evitar usos injustos de estos sistemas, más se enfrenta a grandes dificultades, ya que la realidad en la que incide está en constante transformación, adoptando modalidades, como la mencionada, que buscan evitar la eficacia de las normas por medio de supuestos dudosos, promoviendo la idea de su invalidez por no corresponderse a la realidad.

Es así que, en estos casos grises, resulta positivo analizar las formas en las que la jurisprudencia reciente aborda la problemática, entendiendo que el derecho no se limita simplemente a un entendimiento de las formas jurídicas, sino que pone en juego las construcciones sociales incorporadas por sus actores, quienes inciden, a su vez, en la formación de la realidad (lo que, en términos de Bourdieu constituye una interacción entre habitus y campo). Por lo dicho, es que en los próximos apartados se analizarán las soluciones que la jurisprudencia ha dado a casos que involucran la economía colaborativa, entendiendo cómo se armonizan o no sus sentencias con los conceptos propios del Derecho Laboral, entendiendo como esas sentencias marcan futuros estándares a la hora de regular legalmente estas actividades.

18 KELSEN, HANS. Teoría general de derecho y del estado. Ed. Universidad autonoma de mexico, 1979 


\section{III. ¿Ser "Glover" es trabajar? La relación de dependencia como elemento velado}

En materia jurisprudencial, el abordaje de la economía colaborativa es muy incipiente, centrándose los principales pronunciamientos en nuestro país en la misma pertinencia del fenómeno, siendo el caso más renombrado el fallo de la Corte Suprema de Justicia de la Nación "Uber y otros s/ incidente de recurso extraordinario" del 14 de agosto de 2018, en el cual se desestimó (por motivos procesales) un recurso de queja del Sindicato de Peones de Taxi frente a una sentencia que declaraba a la actividad de Uber como lícita. Ante estas reducidas respuestas, aun teniendo en cuenta la no obligatoriedad de remitirse a la jurisprudencia como fuente del derecho en nuestra legislación, es que resulta útil observar el abordaje que ha tenido la cuestión en la jurisprudencia de otros países, siempre teniendo el recaudo de entender que lo que las mismas resuelven va a estar influido por las situaciones que prevén sus propias legislaciones y dejando presente que aún no hubo sentencias dictadas por Tribunales Supremos, encontrándose la mayoría en situación recursiva.

Con el fin de hacer precisiones de índole general, antes de abordar los casos que hacen a este trabajo, y siguiendo con la situación de Uber, considero oportuno hacer una breve síntesis del que es uno de los primeros pronunciamientos en la materia, el cual marcó las principales líneas a tener en cuenta para considerar el carácter laboral de estas actividades, el fallo "Y. Aslam $y$ J. Farrar $v$. Uber BV, Uber London LTD y Uber Briannia Ltd - case n. 2202551/2015 \& Others» del Employment Tribunals de Londres, Reino Unido. En el caso en cuestión, se exigió que se considera a la relación como un contrato de trabajo, garantizando su protección en el marco legal vigente, situación que la empresa niega alegando que no ofrece servicio de transporte, sino que su función es la de contactar a colaboradores independientes y establecer una serie de pautas mínimas de seguridad. Este planteo resulta rechazado por el tribunal, quien considera 
que la actividad que realiza la empresa Uber LL supone una relación de dependencia, para lo que tiene en cuenta diversos hechos y documentación cuya información puede sintetizarse, según lo señalan Doria y Tosca ${ }^{19}$, en los siguientes puntos: Incorporación a una organización ajena (la plataforma establece las solicitudes a cumplir y los mecanismos básicos para desempeñar la actividad), dependencia económica (en cuanto significa sustento) y control a la persona que trabaja (por medio de un sistema de puntajes, del cual deriva la cuantía del pago que se realiza y la continuidad misma de la relación). A este aspecto, se pueden incorporar otros que resultan relevantes, como lo es la misma calificación de la empresa, la cual alegó ser "una empresa de tecnología", algo que fue desestimado con fundamento en el precedente «Douglas O'Connor v. Uber Technologies Inc.» (11/3/2015), de la Corte de Carolina del Norte, Estados Unidos, considerando que la misma, más allá de la plataforma que usa, lo que ofrece es un servicio de transporte de pasajeros ${ }^{20}$.

Teniendo en claro estos primeros aspectos, y abordando concretamente la aplicación de la economía colaborativa en el "Delivery" o recadeo, la jurisprudencia más relevante a estos fines, tanto por la cercanía idiomática como por la de su espíritu normativo para con el de las leyes laborales argentinas, es la española, en la cual puede destacarse dos casos muy recientes: "Sentencia 244/2018 del Juzgado de lo Social No 6 de Valencia", fechada el día primero de junio de 2018, la cual condenó a la empresa Rappi (empresa inglesa que actúa como competidora directa de Glovo en España, operando en rubros y bajo condiciones de contratación muy similares) por un despido, considerando que medió relación laboral, y «Sentencia 284/2018

19 DORIA, LUCIANO; TOSCA, DIEGO. Alguna aproximación al fenómeno "UBER" y las relaciones laborales. Revista La Causa Laboral N 68, agosto de 2017

20 Este punto y otros considerandos son reseñados en la siguiente nota: https:// aldiaargentina.microjuris.com/2016/12/18/fallos-internacionales-que-marcan-caminos-elcaso-aslam-v-uber-b-v-y-los-derechos-laborales-de-los-choferes-de-uber/. PÉREZ DEL VISO, ADELA. Cita online: MJ-DOC-10586-AR | MJD10586 
del Juzgado de lo Social No 39 de Madrid", con fecha del tres de septiembre de 2018, la cual desestimó la demanda por despido hacia la empresa Glovo, considerando que la contratación realizada no suponía un contrato de trabajo.

La primera sentencia mencionada, la cual responde a la demanda de despido interpuesta por José Enrique frente a ROOFOODS SPAIN S.L.U. (propietarios de la empresa Deliveroo), debiendo resolver primero una excepción de incompetencia impuesta por esta última al considerar que el contrato que celebró con la parte actora no es uno de tipo laboral, sino de prestación de servicios (operando en principio bajo las leyes comerciales y por lo tanto debiendo litigarse en un juzgado de esa materia). Esta primera pretensión es desestimada considerando que en el caso se dan tres puntos que serían esenciales para configurar una relación de trabajo (en base a un análisis del artículo uno del Estatuto de los Trabajadores, principal norma laboral de España), siendo estos la voluntariedad e infungibilidad de la prestación; la ajenidad al momento de realizar los servicios, percibir las ganancias y soportar las pérdidas; y la pertenencia a un ámbito de dirección y organización de otra persona (dependencia). Esta afirmación se logró en base a considerar variedad de elementos que, sin probar manifestaciones tradicionales de estos fenómenos, pueden asimilarse claramente a los mismos si se libran de su ropaje jurídico (en función del denominado "Principio de la primacía de la Realidad"). A lo dicho, y teniendo en cuenta que estos elementos se encuentran presentes en la concepción del Contrato de Trabajo que opera en la Ley de Contrato de Trabajo argentina (ley 20.744), se procederá a analizar su probanza a la luz de las construcciones doctrinarias sobre los mismos.

En primer lugar, la nota de voluntariedad encuentra su fundamento en el mismo hecho de la celebración del contrato por las partes. Este momento del contrato de trabajo ha sido considerado por la doctrina como uno de los pocos en los que opera plenamente la autonomía de la voluntad (elemento propio de toda contratación en el que las partes operan de acuerdo a la 
libertad de contratación para fijar las condiciones que van a regir la relación), siendo que la mayor parte del mismo encuentra su delimitación y regulación en el denominado "orden público laboral ${ }^{21 "}$

En lo que respecta la dependencia, se probó que la demandaba fijaba instrucciones y condiciones unilaterales al momento de llevar adelante la actividad, esencialmente, las de descargar la aplicación, operar en la zona delimitada por el sistema, cumplir las tareas dentro de las franjas horarias establecidas, comenzar el turno en el lugar fijado por la empresa y encontrarse geolocalizado al momento de realizar los repartos.

Finalmente, en lo que depara a la ajenidad, se probó que, a pesar de aportar sus propios elementos para desempeñarse (bicicleta, smartphone), la organización de la actividad empresarial se centraba en una plataforma externa al mismo, la aplicación Deliveroo, desde la cual se fijan, además de las condiciones de la actividad, la propia cuantía de la remuneración obtenida, la cual debía ser abonada a los clientes directamente desde la plataforma para luego ser esta misma quien elabore la factura del percibido por la parte actora. Además, se establecía la imposibilidad de rechazar pedidos ya aceptados, con excepción de encontrar a otro trabajador/a que se dispusiera a aceptarlos.

Ante todo lo dicho, y sin que la demandada pudiera desandar las afirmaciones anteriores, el tribunal consideró que la relación era de carácter laboral, receptando por lo tanto que las comunicaciones en las que la parte actora se consideró injuriada y despedida, permitían comprender a la extinción de la relación como un despido indirecto, a lo que procedió a rechazar la excepción de incompetencia y condenar a la demandada a readmitir al trabajador o indemnizar lo daños que le generó esta situación.

21 Defínase como "el conjunto de normas imperativas que se imponen a la voluntad de las partes, en tanto éstas no establezcan condiciones más favorables al trabajador". FERNÁNDEZ MADRID, JUAN CARLOS. Tratado Práctico de Derecho del Trabajo. Tomo I. Ed. La Ley, 2007. Pag. 590 


\section{EI triunfo de la forma: Divergencias en la apreciación judicial de la relación}

Como se señaló previamente, el abordaje de esta cuestión es muy incipiente e implica analizar fenómenos cuyas implicancias se alteran fuertemente según el punto de vista que se adopte, por lo mismo, no es extraño que un par de meses después de dictarse la sentencia anterior, específicamente en fecha 3 de septiembre, otro tribunal español, el Juzgado de los Social $N^{\circ} 39$ de Madrid, haya dictado una sentencia que tuviera gran divergencia apreciativa sobre hechos muy similares a la anterior, llevando a desestimar una demanda por despido indirecto formulada por un antiguo Glover hacia la empresa en la que se desempeñaba. Frente a esta situación, procederemos a contrastar lo resuelto en este caso con respecto a lo que se apreció en el anterior.

En este caso, se puede señalar en primer lugar que la desestimación de la demanda tuvo fundamentos que exceden a la mera consideración de la actividad como una prestación de servicios, ya que la forma en la que se desarrolló en intercambio telegráfico previo generó conflictos al momento de apreciar las fechas de los hechos alegados, lo cual afectó fuertemente la consideración de los mismos. No obstante lo dicho, el punto interesante en esta jurisprudencia se encuentra al momento de considerar la naturaleza del contrato celebrado, al que la jueza en cuestión le atribuye el carácter de una prestación de servicios, como alegaba la parte demandada (Glovo), resultando relevante su apreciación ya que, a pesar de resolver desfavorablemente, la misma no discute que este tipo de casos deben resolverse en función del Principio de Primacía de la realidad.

En primer lugar, debido a que van a ser las bases en las que se apoye la sentencia, hay que tener en cuenta que la noción que se utiliza en este caso para considerar los conceptos de dependencia y ajenidad se apoya en ideas muy tradicionales sobre estos, con un corte arraigado a la actividad fabril, estableciendo desde un principio que los indicios que distingue para 
caracterizarlos son para el primer caso: la asistencia al centro de trabajo del empleador, el sometimiento a un horario y la inserción del trabajador en una estructura ajena (con la consiguiente ausencia de organización empresarial propia), y para el segundo: La puesta a disposición de los productos realizados, la adopción por el empresario de las decisiones propias del mercado (como la fijación de precios), el carácter periódico de la remuneración y la adopción de un criterio de proporcionalidad entre salario y cantidad de actividad realizada.

Ya abordando concretamente lo dispuesto en el fallo, lo primero a tener presente es que, según se sostiene, la única nota de las mencionadas para configurar un contrato de trabajo según el artículo 1 del Estatuto, es la de la voluntariedad, la cual es propia de todo tipo de contrato. Establecido esto, debe tenerse presente que, el principal argumento que se utiliza para cuestionar la presencia de dependencia y ajenidad está vinculado a que el contrato de prestación de servicios celebrado fue hecho bajo la modalidad TRADE ${ }^{22}$ (Trabajador Autónomo Dependiente Económicamente), una figura incorporada por la ley española 20/2007, denominada comúnmente "Estatuto del Trabajador Autónomo", que implica la posibilidad de que una persona que a nivel formal figure como autónomo, en la práctica realice actividades y apoye la mayoría de su sustento en otra persona, con la que supuestamente operaría en igualdad de condiciones. La jueza se apoya fuertemente en el contenido que figura en estos contratos, los cuales estipulan que: el repartidor es el que organiza su trabajo, que responde por los daños ocasionados durante el transporte, que su retribución depende de la cantidad de recados realizados, que la realización de los trabajos se realiza bajo las pautas del propio cliente (operando Glovo sólo como un intermediador que pone a disposición estos trabajos),

22 La ley 20/2007 los define en su artículo 11, inciso 1 como “(...) aquéllos que realizan una actividad económica o profesional a título lucrativo y de forma habitual, personal, directa y predominante para una persona física o jurídica, denominada cliente, del que dependen económicamente por percibir de él, al menos, el 75 por ciento de sus ingresos por rendimientos de trabajo y de actividades económicas o profesionales". 
las principales herramientas de trabajo son proporcionadas por el trabajador/a y el pago será el establecido en las tarifas adjuntadas por Glovo, quien elaborará factura por plazos de 15 días.

Al considerar que no hay prueba presentada que desvirtúe los dichos de esos contratos (habiéndose desestimado un testimonio de otro ex trabajador en conflicto con la empresa), se procede a verificar si la situación que describen puede configurar una relación laboral. A lo dicho, se pone en consideración que el trabajador no tenía jornada ni horario, elegía en que franja horaria trabajar, elegía con libertad que pedidos hacer (pudiendo cancelarlos en medio del mismo sin recibir penalizaciones), que ruta tomar para llegar a destino y que las instrucciones sobre el pedido eran establecidas por el consumidor destinatario. Ante lo dicho, el tribunal sostiene que "Ninguna de estas características son predicables en una relación laboral ordinaria, donde el trabajador está sujeto a una jornada y horario, debe realizar las actividades encomendadas sin poder elegir cuáles hacer y cuáles no, debe seguir el método de trabajo impuesto por la empresa (...)".

De lo dicho ya se pueden avizorar grandes divergencias en cuanto a la forma de abordar hechos similares, ya que, a pesar de haber diferencias entre las prácticas de ambas empresas (por ejemplo, la posibilidad de rechazar pedidos en medio de su realización, la cual cuenta con muchas más facilidades en Glovo), hay situaciones que se reconocen en ambos casos y que son asumidas sin cuestionamiento en esta sentencia, como lo es la delimitación de franjas horarias y destinos/pedidos realizada por la empresa a través de la aplicación de teléfono, lo cual acota la libertad de operación que se propugna - el fallo sostiene que el régimen TRADE permite proporcionar "ciertas indicaciones técnicas", lo cual, en este caso, puede cuestionarse, ya que, sin esta estructura no podría realizarse la actividad-. Por otro lado, resulta discutible sostener como una señal de independencia el hecho de que sean los clientes quienes dispongan el pedido, ya que eso pondría en cuestionamiento la laboralidad de cualquier actividad de envío a domicilio de productos. 
Posteriormente, la sentencia procede a analizar la existencia del poder disciplinario de la empleadora, sosteniendo que, al poseer mecanismos muy acotados, se ratifica la capacidad autoorganizativa del trabajador. Menciona esencialmente dos aspectos: La posibilidad de desistimiento del contrato en caso de no realizar actividad (la cual, como se señala, es una característica propia de cualquier contrato) y un sistema de puntuación, al cual no valora de esa forma, ya que considera que no implica situaciones sancionatorias, sino una escala preferencial al momento de acceder a pedidos (mayor cantidad disponible al tener más puntos), lo cual no sería discutido, sosteniendo que "premiar a unos trabajadores por la superior calidad o cantidad del trabajo no equivale a castigar a los demás". Sostener este punto de vista implica naturalizar un sistema que claramente establece una superioridad de la empresa con respecto a los trabajadores/as, habilitando a que administre la accesibilidad al empleo de los mismos, lo cual se ratifica al ver que estos puntajes pueden ser incluso rebajados por la empresa al no presentarse a una franja horaria previamente reservada, no resultando relevante la posibilidad de apelar o justificar la sanción, ya que esto también es una posibilidad contemplada en las leyes laborales (como se puede atestiguar en la regulación de las facultades disciplinarias del empleador que se encuentran en el artículo 67 de la Ley de Contrato de Trabajo argentina y 58 del Estatuto de los Trabajadores español).

Un último aspecto a considerar es lo que refiere a la percepción del salario, ya que la sentencia sostiene que en un régimen TRADE se está facultado para "señalar el precio de los servicios" - aspecto que haría que, para estos contratos, el que el empleador posea esta facultad no sea una nota propia de relación laboral -, aun así, para el caso en concreto, también se estaría en incumplimiento, ya que la empresa establece condiciones de facturación y percepción de la contraprestación que colocan en una situación de ajenidad al trabajador en cuestión. 


\section{Conclusiones: Una disputa que ya comenzó}

Más allá de la ya mencionada divergencia en las apreciaciones judiciales, y el riesgo desprotectorio que puede suponer la figura del TRADE, la última sentencia analizada resulta muy relevante porque deja entrever una realidad cada vez más patente en el mercado de trabajo: Su acelerada movilidad y transformación producto de las influencias tecnológicas. El avance en las tecnologías de la información ha introducido gran variedad de dinámicas novedosas en las relaciones sociales, por lo que no incorporar estas cuestiones a un análisis sobre el mundo del trabajo supone una visión restrictiva y sumamente desprotectoria para un sector de la población que debe ser visto (según lo expresado por la Corte Suprema de Justicia de la Nación) como "sujeto de preferente tutela constitucional23", ya que, como menciona Méndez ${ }^{24}$, "el ordenamiento jurídico es producto de una realidad histórica y sociocultural".

No reconocer carácter laboral a las relaciones que se originan en la economía colaborativa supone dejar en una situación de marginalidad a un sector de las trabajadoras/es que, a pesar de su carácter incipiente, se proyecta y crece con cada vez más velocidad. Este hecho es muy marcado en Argentina, donde, a pesar de la falta de información oficial, se estima que se encuentran operando aproximadamente trece mil trabajadores/as de Rappi (con principal radicación en Capital Federal) y cinco mil de Glovo $^{25}$ (quienes realizan una expansión a nivel nacional, en provincias como Buenos Aires, Santa Fe y Córdoba), lo cual, sumado a la reciente revelación que hizo Uber - dejando constancia de la incorporación de cincuenta y cinco mil empleadas/os

\footnotetext{
23 Referenciado en numerosos fallos del tribunal, destaquese "Vizzoti c/ Amsa" y "Aquino c/ Cargo S.A."

24 MÉNDEZ, ELISA. "Las ideologías y el derecho", Ed. Astrea Buenos Aires 1982

25 https://www.bigbangnews.com/politica/Gremio-low-cost-trabajadores-de-RappiGlovo-y-UBER-se-aliaron-para-crear-un-sindicato-20181010-0014.html
} 
en el periodo Julio - Septiembre de $2018^{26}$ - deja claro la urgente necesidad de regular estas actividades.

Teniendo en claro esta problemática, debe señalarse que las preocupaciones que la misma genera no se han quedado limitadas a pronunciamientos públicos, sino que, lentamente, va configurándose un marco de acción, que puede expresarse esencialmente en dos planos: La recepción y recomendación normativa desde la OIT y la realización de protestas y expresiones sindicales desde quienes trabajan para estas empresas.

En primer lugar, como se señaló previamente, la OIT expresó su preocupación por el futuro del trabajo, la cual llevó que en agosto de 2017 se dispusiera la creación de una Comisión Mundial sobre el Futuro del Trabajo en el marco de la misma, compuesta por seis grupos temáticos que elaboraron notas informativas de situación publicadas en febrero de $2018^{27}$, siendo relevantes para este trabajo las del Grupo 3 (Tecnología al servicio del desarrollo social, ambiental y económico). La nota "La influencia de la tecnología en la calidad y la cantidad del empleo" aporta datos estadísticos que permiten entrever que el impacto de la digitalización va a producir a largo plazo una destrucción, pero a la vez un surgimiento de nuevos puestos en el mercado de trabajo, generando, entre otros interrogantes, el pensar como acercar los beneficios tecnológicos a los países en desarrollo. Por su parte, la nota "La calidad del trabajo en la economía de plataformas" resulta un material muy importante e innovador en lo que refiere al abordaje normativo de la cuestión, dejando patente el reconocimiento de la condición laboral de quienes prestan los servicios, elaborando estadísticas, señalando sus ventajas (flexibilidad en en el desempeño de la actividad laboral y reducción de costos empresariales) e inconvenientes (no reconocimiento de la relación laboral, escasa remuneración y dificultades al momento de garantizar

26 https://www.infobae.com/economia/2018/09/25/argentina-registro-el-mayorcrecimiento-de-uber-en-todo-el-mundo/

27 https://www.ilo.org/global/topics/future-of-work/WCMS_618595/lang--en/index.htm 
seguridad social), e instala varias inquietudes que pueden servir de lineamientos para la elaboración de legislación.

Delimitándose al ámbito del continente americano, la actividad de la OIT en este aspecto ha sido más acotada, pero no por eso menos relevante, realizándose entre el 2 y el 5 de Octubre de 2018, en Panamá la Decimonovena Reunión Regional Americana la cual dio lugar a la llamada "Declaración de Panamá para el centenario de la OIT: por el futuro del trabajo en las Américas"28, un documento que señala prioridades a seguir en políticas de empleo comunes a todos los países y líneas de acción que la OIT debe desempeñar para su concreción. Dentro de las prioridades mencionadas, es destacable el punto décimo, el cual refiere a establecer "las políticas para responder a las nuevas y diversas formas de empleo creadas por las revoluciones tecnológicas y los nuevos modelos de negocios", llamando a "eliminar vacíos normativos, fortalecer la negociación colectiva, la protección social y las políticas de empleo de calidad" y favoreciendo, a su vez, "la creación, mantenimiento y desarrollo de empresas". El texto elaborado, aunque impreciso (debido a los conflictos que implicó acordar entre sectores laborales, empresariales y estatales $^{29}$ ), resulta un primer gran basamento en lo que refiere a reconocer esta problemática y generar la obligación de regularizarla en los ámbitos normativos internos.

Por último, resulta destacable el rol que tuvo la organización y protesta de quienes trabajan en estos espacios, la cual es sin dudas la base de todos los reclamos y declaraciones que se llevaron adelante, permitiendo visibilizar la problemática e incluso motivando los reclamos judiciales analizados ${ }^{30}$. Recientemente se han realizado múltiples huelgas y movilizaciones, entre las

28 Disponible en: https://www.ilo.org/wcmsp5/groups/public/@ed_norm/@relconf/documents/meetingdocument/wcms_646374.pdf

29 En línea en: https://www.iprofesional.com/economia/279361-delivery-cgt-sindicatosUber-Rappi-y-Glovo-en-la-mira-sindicalistas-y-empresarios-se-cruzaron-en-OIT-por-elimpacto-laboral-de-las-app

30 Entrevista a Isaac Cuende, quien promovió la demanda a Glovo que se abordó en el artículo: https://www.eldiario.es/economia/mensajero-Glovo-tribunales-demostrar-autonomos_0_791970972.html 
que pueden destacarse la realizada en Zaragoza, España (la cual consistió en no atender los reclamos solicitados por la aplicación en una franja horaria ${ }^{31}$ ), replicándose en otras ciudades gradualmente ${ }^{32}$, la concentración de trabajadores/as de Rappi frente a sus oficinas en Capital Federal ${ }^{33}$ (primera huelga de este tipo en en el país) y la convocatoria que realizaron quienes trabajan en Glovo en La Plata ${ }^{34}$, la cual dio lugar a un comunicado colectivo en que se denunció la situación de precariedad ${ }^{35}$. A su vez, se han desarrollado incipientes actividades sindicales, tanto desde la ASIMM, como a través de la presentación para inscripción de un sindicato que represente a quienes realicen actividad por plataformas virtuales, la Asociación de Personal de Plataformas ${ }^{36}$.

Estas acciones resultan, sin duda, un ejemplo sobre el cómo defender el derecho a que estos nuevos trabajos, muy necesarios en un momento de crisis en el acceso al empleo, puedan ejercerse sin someterse a condiciones de desamparo y explotación, pudiendo concluir desde un ámbito jurídico que, como expuso Ghersi ${ }^{37}$, "Debemos entonces cuestionar estos límites meramente economicistas, sin legitimidad, más que la natural lógica del mercado, inaplicable a cuestiones sociales fundantes. Recordar

31 En línea en: https://www.elconfidencial.com/tecnologia/2018-09-10/glovo-paralizanzaragoza-riders-precariedad_1613740/

32 https://www.economiadigital.es/directivos-y-empresas/los-repartidores-de-glovobuscan-un-colapso-nacional_577763_102.html

33 https://www.iprofesional.com/tecnologia/271673-internet-salario-argentina-Primerahuelga-del-delivery-online-en-la-Argentina-denuncian-cambios-unilaterales-en-las-condiciones-laborales

34 http://infoblancosobrenegro.com/noticias/22010-los-repartidores-de-glovo-en-la-platacomenzaron-a-organizarse-y-preparan-un-plan-de-lucha

35 https://www.0221.com.ar/nota/2018-9-24-10-20-0-abandono-e-inestabilidad-el-fuertereclamo-de-los-trabajadores-de-glovo-en-la-plata

36 https://www.perfil.com/noticias/innovacion/la-economia-de-las-plataformas-ya-tienesu-primer-sindicato-en-el-pais.phtml

37 GHERSI, CARLOS ALBERTO. Los límites del derecho como orden social justo (Reflexiones para la posmodernidad jurídica). Revista Lecciones y Ensayos Nº63 (1995). Facultad de Derecho. Universidad de Buenos Aires. 
que el centro del Derecho es el ser humano, y no el mercado y que las interrelaciones entre aquéllos - la intersubjetividad de Cossio - sólo pueden bacerse en un marco de ética, equidad y justicia”.

\section{Bibliografía y fuentes}

BOURDIEU, PIERRE; TEUBNER, GUNTHER. La fuerza del derecho. Bogotá: Siglo del Hombre Editores. Facultad de Derecho de la Universidad de los Andes, Ediciones Uniandes, Instituto Pensar. 2000.

DE HEREDIA RUÍZ, IGNASÍ BELTRÁN - Caso Glovo: ¿los repartidores no son trabajadores por cuenta ajena? (análisis crítico a la SJS/39 Madrid de 3/9/18). Artículo de opinión, disponible en: https://ignasibeltran.com/2018/09/20/caso-glovo-losrepartidores-no-son-trabajadores-por-cuenta-ajena-analisiscritico-a-la-sjs-39-de-3-9-18/

DOMÉNECH PASCUAL, GABRIEL. La regulación de la economía colaborativa (El caso Uber contra Taxi). REVISTA CEFLEGAL. CEF, núms. 175-176 (agosto-septiembre 2015) - Pag. 67. Disponible en: http://roderic.uv.es/handle/10550/54302

DORIA, LUCIANO; TOSCA, DIEGO. Alguna aproximación al fenómeno "UBER" y las relaciones laborales. Revista La Causa Laboral $\mathrm{N}^{\circ}$ 68, agosto de 2017. Disponible en: http://www. laboralistas.net/articulo_revista/alguna-aproximacion-al-fenomeno-uber-y-relaciones-laborales/

FERNÁNDEZ MADRID, JUAN CARLOS. Tratado Práctico de Derecho del Trabajo. Tomo I. Ed. La Ley, 2007.

GATTI, ÁNGEL EDUARDO. Derecho del trabajo. Manual de las relaciones individuales. Ed. B de F, 2015

GHERSI, CARLOS ALBERTO. Los límites del derecho como orden social justo (Reflexiones para la posmodernidad jurídica). Revista Lecciones y Ensayos No 63 (1995). Facultad de Derecho. Universidad de Buenos Aires.

LOZANO, MARÍA PAULA; CASIRAGHI, FEDERICO. Tercerización, precarización y solidaridad en las relaciones laborales. La Ley Online. Cita Online: AR/DOC/1477/2013 
ORSINI, JUAN IGNACIO. Los Principios del Derecho del Trabajo. Revista Anales de la Facultad de Ciencias Jurídicas y Sociales; año 7, no. 40. Disponible en: http://sedici.unlp.edu. ar/bitstream/handle/10915/21014/Documento_completo. pdf? sequence $=1$

PACHECO JIMÉNEZ, MARÍA NIEVES. La Web 2.0 como instrumento esencial en la economía colaborativa: Auge de negocios de dudosa legalidad. Publicado en Revista CESCO de Derechos de Consumo $N^{\circ} 17 / 2016$ - Universidad de Castilla y La Mancha, España. Disponible en: https://www.revista.uclm.es/index. $\mathrm{php} / \mathrm{cesco} /$ article/view/1055

PÉREZ DEL VISO, ADELA. Fallos internacionales que marcan caminos: El caso "Aslam v. Uber B.V." y los derechos laborales de los choferes de Uber. Cita online: MJ-DOC-10586-AR | MJD10586. Disponible en:

PETORUTTI, CARLOS ENRIQUE; SCATOLINI, JULIO CÉSAR. Elementos de Introducción al Derecho. Ed. La Ley. 2007.

VALLECILLO GÁMEZ, MARÍA ROSA. Economía colaborativa y laboralidad: Los cabos sueltos entre el vacío legal y la dudosa legalidad. Propuesta para Conversación 3 sobre El futuro del trabajo organizada por OIT - Universidad de Jaén. 